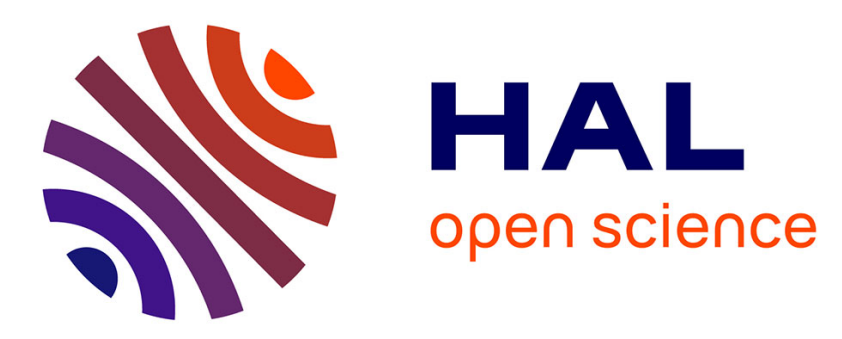

\title{
Blind Navigation of Web Pages through Vibro-tactile Feedbacks
}

Waseem Safi, Fabrice Maurel, Jean-Marc Routoure, Pierre Beust, Michèle Molina, Coralie Sann, Jessica Guilbert

\section{To cite this version:}

Waseem Safi, Fabrice Maurel, Jean-Marc Routoure, Pierre Beust, Michèle Molina, et al.. Blind Navigation of Web Pages through Vibro-tactile Feedbacks. 25th ACM Symposium on Virtual Reality Software and Technology (VRST 2019), Nov 2019, Sydney, Australia. 10.1145/3359996.3364758 . hal-02309657

\section{HAL Id: hal-02309657 https://hal.science/hal-02309657}

Submitted on 9 Oct 2019

HAL is a multi-disciplinary open access archive for the deposit and dissemination of scientific research documents, whether they are published or not. The documents may come from teaching and research institutions in France or abroad, or from public or private research centers.
L'archive ouverte pluridisciplinaire HAL, est destinée au dépôt et à la diffusion de documents scientifiques de niveau recherche, publiés ou non, émanant des établissements d'enseignement et de recherche français ou étrangers, des laboratoires publics ou privés. 


\section{Blind Navigation of Web Pages through Vibro-tactile Feedbacks}

\author{
Waseem Safi \\ waseem.safi@unicaen.fr \\ University of Caen Normandy \\ Caen, France \\ Pierre Beust \\ pierre.beust@unicaen.fr \\ University of Caen Normandy \\ Caen, France
}

\author{
Fabrice Maurel \\ fabrice.maurel@unicaen.fr \\ University of Caen Normandy \\ Caen, France \\ Michèle Molina \\ michele.molina@unicaen.fr \\ University of Caen Normandy \\ Caen, France \\ Jessica Guilbert \\ jessica.guilbert@unicaen.fr \\ University of Caen Normandy \\ Caen, France
}

\author{
Jean-Marc Routoure \\ jean-marc.routoure@unicaen.fr \\ University of Caen Normandy \\ Caen, France \\ Coralie Sann \\ coralie.sann@unicaen.fr \\ University of Caen Normandy \\ Caen, France
}

\begin{abstract}
We present results of an empirical study for examining the performance of sighted and blind individuals in discriminating structures of web pages through vibro-tactile feedbacks.
\end{abstract}

\section{CCS CONCEPTS}

- Human-centered computing $\rightarrow$ Human computer interaction (HCI); Graphical user interfaces.

\section{KEYWORDS}

Vibro-tactile feedbacks, visually impaired users, accessibility

\section{ACM Reference Format:}

Waseem Safi, Fabrice Maurel, Jean-Marc Routoure, Pierre Beust, Michèle Molina, Coralie Sann, and Jessica Guilbert. 2019. Blind Navigation of Web Pages through Vibro-tactile Feedbacks. In 25th ACM Symposium on Virtual Reality Software and Technology (VRST '19), November 12-15, 2019, Parramatta, NSW, Australia. ACM, New York, NY, USA, 2 pages. https: //doi.org/10.1145/3359996.3364758

\section{INTRODUCTION}

This work has been realized by designing a tactile vision sensory substitution system (TVSS). The paper is organized as following: in section 2, the related works are presented; in Section 3, the realized vibro-tactile framework is introduced. In section 4 , the protocol of the experiment is described. Finally, the experiment results and the conclusion are given in the last sections.

Permission to make digital or hard copies of all or part of this work for personal or classroom use is granted without fee provided that copies are not made or distributed for profit or commercial advantage and that copies bear this notice and the full citation on the first page. Copyrights for components of this work owned by others than ACM must be honored. Abstracting with credit is permitted. To copy otherwise, or republish to post on servers or to redistribute to lists, requires prior specific permission and/or a fee. Request permissions from permissions@acm.org.

VRST '19, November 12-15, 2019, Parramatta, NSW, Australia

(c) 2019 Association for Computing Machinery.

ACM ISBN 978-1-4503-7001-1/19/11 \$ \$15.00

https://doi.org/10.1145/3359996.3364758

\section{RELATED WORKS}

Researches in the field of vibro-tactile interactions begin as early as the 1920 s, with a system that tested the feasibility of transferring speech into vibro-tactile stimuli [2]. Opticon is one of oldest systems that proposed a vibro-tactile feedback [1] . A rich tactile output system has been proposed to evaluate the potential of rich tactile notifications on mobile phones [5]. Tactos is a perceptual interaction system [3] that consists in tactile simulators, a graphics tablet with a stylus, and a computer [6]. The main drawback of some proposed systems is that they are not oriented for web navigation, and they have been designed to present basic shapes.

\section{VIBRO-TACTILE FRAMEWORK}

The designed system "TactiNET" (cf. figure 1) provides one pattern vibro-tactile feedback when the blind user touches a tablet. We have designed an electronic circuit, which controls many micro-vibrators placed anywhere on the body. A Bluetooth connection with an android tablet allows controlling the actuators. The intensity of the light emitted by the tablet at touched points is then transmitted to the embedded device in order to control the tactile stimuli.

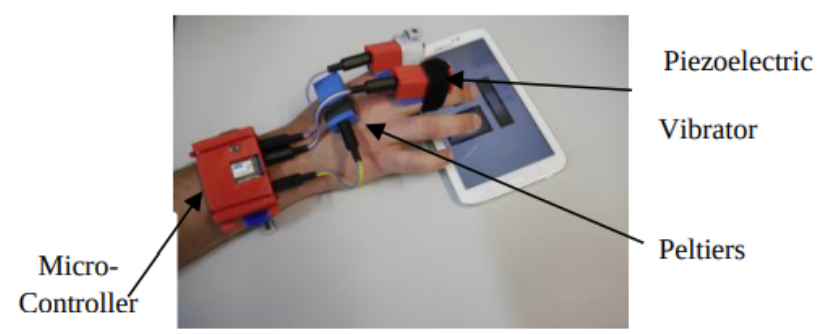

Figure 1: “TactiNET” prototype.

TactiNET provides communication between an electronic device (base device) and an array of 8 satellites that control 8 different actuators. Each satellite is connected to the main device via a cable that provides both power and communication. 


\section{PROTOCOL OF THE EXPERIMENT}

This experiment is based on converting semi-automatically the visual structures of web pages into vibrating pages. Representing the shapes as rectangles has been chosen depending on a series of experiments with sighted persons to understand how they segment web pages; and after running an empirical experiment with blind persons to examine their performance in recognizing rectangular shapes through vibro-tactile feedbacks [4]. Three representative web pages have been chosen to be evaluated in this experiment.

An agglomerative graph-based clustering algorithm has been applied on each representative page. Its output is a graph of $\mathrm{N}$ segments (clusters), where each cluster contains similar (close) HTML elements. In this experiment, the number of clusters for each web page has been chosen to be equal 5. This option has been chosen depending on the theory of the magic number seven plus or minus two (Miller's Law).

A particular vibro-tactile feedback should be dedicated for each shape. These vibro-tactile feedbacks could be varied in frequency, amplitude, waveform, and duration. The designed device can control only the frequency and the amplitude values of the used actuators. So the contents of all the graphical forms will be represented in vibration mode by controlling frequency and amplitude parameters. In order to represent the vibration feedback dedicated for each segment, the standard deviation value calculated for each segment has been assigned (manually) to the amplitude value (amplitude=SD), and a constant value $304.6875 \mathrm{~Hz}$ has been assigned to the frequency. We have used another constant value of amplitude equals to 55 in order to evaluate if there is a difference in performance when using fixed or variant values of amplitudes [4].

Each experiment (either with sighted or blind persons) consists of a series of tests to navigate two touched-screen tablets, and to compare the web pages' structures presented on the two tablets, then to decide if the two navigated web pages' structures are identical or not. The participant navigates the first tablet with one of his/her index fingers, and puts the another index finger on the actuator to perceive the vibrations. The participant then navigates the second tablet with one of his/her index fingers, and puts the another index finger on the actuator to perceive the vibrations. After navigating the two devices, the participant decides if the two structures are identical or not, and then starts redrawing the discovered web pages' structures on A4 paper. 36 tests of structures comparison have been achieved by each participant. Considering that for each comparison, the experimenter tests two amplitude values (variant values, and fixed value 55).

11 persons have participated in this experiment. 5 of them are sighted, and 6 are blind. The 6 blind participants ( 3 males and 3 females) are different in the date of their vision loss. 3 of them have a congenital vision loss (since birth), and the other 3 participants have adventitious vision loss (after birth). The average of ages for the blind participants is 54.8 years. 5 of them have participated in previous pre-test experiments. All the sighted participants are females. Their average of ages is 26.4 years.

\section{ANALYSIS OF RESULTS}

Each participant has answered 36 questions about the similarity or dissimilarity of two navigated structures. The proposed question is "can you indicate, as quick as possible, if the two spatial structures are identical?". In what concerns the blind participants: for the fixed amplitude (55), the percentage of correct answers while comparing similar structures is $40,7 \%$, and $81,5 \%$ for dissimilar structures. For the variable amplitude (SD values), the percentage of correct answers while comparing similar structures is $50,0 \%$, and $79,6 \%$ when comparing dissimilar structures.

In what concerns the sighted participants: for the fixed amplitude (55), the percentage of correct answers while comparing similar structures is $37,8 \%$, and $84,4 \%$ when comparing dissimilar structures. For the variable amplitude (SD values), the percentage of correct answers while comparing similar structures is $37,8 \%$, and $84,44 \%$ when comparing dissimilar structures. By analyzing the presented data, the following results could be concluded: 1) there is not a remarkable difference in discriminating the structures between the blind and the sighted participants. 2) concerning the sighted participants, there is not a remarkable difference in recognizing the structures by changing the amplitude values.

\section{CONCLUSION AND PERSPECTIVES}

A non-visual interaction approach has been presented in this work. The approach is based on a vibro-tactile modality. Training the participants on the designed system for a sufficient period enhances their performance and their ability to recognize the navigated structures.

Training the blind participants to navigate the Web using touchscreen devices enhances their ability to deal with the designed system. Analyzing many parameters concerning the global and the micro navigation strategies such the time, the speed, and their successive might result new observations. These observations could be useful in developing new versions of the designed system.

\section{REFERENCES}

[1] James C. Bliss, Michael H. Katcher, Charles H. Rogers, and Raymond P. Shepard. 1970. Optical-to-Tactile Image Conversion for the Blind. In Man-Machine Systems, Vol. 11. IEEE, 58-65. https://doi.org/10.1109/TMMS.1970.299963

[2] Seungmoon Choi and Katherine J. Kuchenbecke. 2013. Vibrotactile Display: Perception, Technology, and Applications. In Proceedings of the IEEE, Vol. 101. IEEE, 2093-2104. https://doi.org/10.1109/JPROC.2012.2221071

[3] Yvette Hatwell, Arlette Streri, and Edouard Gentaz. 2003. Touching for Knowing. In The tactile reading of maps and drawings, and the access of blind people to works of art. John Benjamins Publishing Company, 255-273.

[4] Waseem Safi, Fabrice Maurel, Jean-Marc Routoure, Pierre Beust Michèle Molina, Coralie Sann, and Jessica Guilbert. 2017. Which Ranges of Intensities Are More Perceptible for Non-Visual Vibro-Tactile Navigation On Touch-Screen Devices. In proceedings of the 23rd ACM Symposium on Virtual Reality Software and Technology (VRST2017). ACM New York, NY, USA ( 2013 . https://doi.org/10.1145/3139131. 314122

[5] Alireza Sahami, Paul Holleis, Albrecht Schmidt, and Jonna Häkkilä. 2008. Rich Tactile Output on Mobile Devices. In Proceedings of Ami'08 European Conference on Ambient Intelligence. Springer Verlag, 210-221. https://doi.org/10.1007/978-3540-89617-3_14

[6] Matthieu Tixier, Charles Lenay, Gabrielle Le Bihan, Olivier Gapenne, and Dominique Aubert. 2013. Designing Interactive Content with Blind Users for a Perceptual Supplementation System. In Proceedings of the 7th International Conference on Tangible, Embedded and Embodied Interaction. ACM New York, NY, USA (c)2017, 229-236. https://doi.org/10.1145/2460625.2460663 